\title{
Conflictos armados y cobertura mediática: aproximación al aprendizaje de máquina supervisado
}

\author{
Armed conflicts and Media Coverage: \\ Supervised Machine Learning Approach
}

José Manuel Moreno-Mercado (D) https://orcid.org/0000-0003-0067-5051

Universidad de Granada, España,jmmoreno95@correo.ugr.es

Javier García-Marín (D) https://orcid.org/0000-0002-2766-0266

Universidad de Granada, España,jgmarin@ugr.es

\begin{abstract}
The media coverage of armed conflicts tends to focus on the attribution of responsibilities and the humanitarian or security frames, as the main studies about media and conflicts have asserted. The present research analyzes the frames used in media outlets such as El Mundo and El Pais (Spain), HispanTV (Iran) and RT (Russia), in their coverage of wars in Yemen and Ukraine. Through innovative and machine learning techniques (particularly, SVM), the authors demonstrate that RT and HispanTV do not follow the traditional routines of mass media, and that the use of frames rely more on the geostrategic position of their countries of origin.
\end{abstract}

Key words: frames, mass media, conflicts, Ukraine, Yemen, SVM.

Resumen: La cobertura mediática de los conflictos armados suele centrarse en la atribución de responsabilidades, las consecuencias humanitarias o la seguridad, como señalan los principales estudios sobre medios y conflictos. La presente investigación analiza el uso de encuadres en los medios El Mundo, El País (España), HispanTV (Irán) y RT (Rusia) cuando cubren las guerras de Yemen y Ucrania. A través de novedosas técnicas de aprendizaje de máquina (en concreto, SVM), se muestra que los medios ruso e iraní no siguen las pautas de las rutinas periodísticas tradicionales, sino que el uso de encuadres depende de la posición geoestratégica de sus países de origen.

Palabras clave: encuadres, medios de comunicación, conflictos, Ucrania, Yemen, SVM. 


\section{Introducción}

Los terribles y desgarradores sucesos acaecidos en Ruanda (1994), Bosnia (1995) y Kosovo (1999) aportaron nuevos conceptos asociados a la seguridad, como los de seguridad humana o responsabilidad de proteger, que, sin duda, también se han exportado a la cobertura realizada por los medios de comunicación, como lo hicieron, en los ochenta, las hambrunas en algunas zonas de África. Así, el comportamiento de la prensa cambia dependiendo del contexto informativo, como en tiempos de paz frente a tiempos de guerra (García-Marín y García-Luengo, 2009: 367). Igual sucede cuando los medios encuadran la realidad de la política interior frente a la exterior (Canel, 1999). Quizá la mejor herramienta que describe estos comportamientos sea los encuadres (frames). La construcción de encuadres, entonces, fluctúa dentro de unos esquemas explicativos genéricos cuando se abordan conflictos armados, como señalaban las investigaciones de Semetko y Valkenburg (2000), y a su vez se deslindan en otros específicos, amoldados a las características del conflicto en particular.

En este sentido, la cobertura mediática de los conflictos armados ha arrojado aportaciones novedosas sobre el uso de encuadres en el presente milenio. Especialmente significativo ha sido el uso de los encuadres guerra étnica y genocidio en el reporte de noticias sobre Oriente Medio en la Posguerra Fría (Hammond, 2018: 434).

Los actuales conflictos de Yemen y Ucrania son en particular interesantes desde el punto de vista de la comunicación política por varias razones. En primer lugar, por la enorme cobertura mediática que han recibido por parte de todos los medios de comunicación del mundo. Y, en segundo lugar, por la implicación directa o indirecta de grandes potencias, potencias regionales, organizaciones no gubernamentales y actores no estatales que conforman la comunidad internacional. Ambos conflictos han suscitado una gran cantidad de literatura académica desde enfoques multidisciplinares, como el derecho internacional (Hillier y Cairns, 2018), la comunicación (MacDonald, 2018) o la ciencia política (Zabyelina, 2019).

En virtud de ello, es también singular la relevancia que ha tenido la irrupción de nuevos medios de comunicación, con formatos en múltiples idiomas, como RT o HispanTV a la hora de cubrir episodios bélicos en los últimos años. Las teorías que relacionan el poder blando con las nuevas dinámicas estratégicas de algunos países, como la Federación Rusa, ${ }^{1}$ inducen a

1 Es especialmente conocida la llamada Doctrina Gerasimov, aunque no existe consenso entre los autores sobre sus fines y características (Gerasimov, 2013). 
preguntarse si podemos equiparar estos medios a los medios de comunicación tradicionales en entornos democráticos. Máxime cuando estos países son considerados como enemigos de la información por importantes organizaciones internacionales, como Reporteros Sin Fronteras. ${ }^{2}$

Como señala la literatura especializada, el uso de encuadres durante el desarrollo de conflictos armados, independientemente de la polarización o indexación ideológica, se desarrolla bajo unos esquemas muy delimitados, como en el caso sirio (Moreno-Mercado, 2018). Premisa que si analizamos en los escasos estudios que abordan el fenómeno de estos medios, induce a afirmar lo contrario (Yablokov, 2015; Peña-Ramos y Jorquera-Mery, 2017). De ahí que esta investigación cobre sentido tanto para abordar un fenómeno escasamente tratado, como para sentar cierta base teórica al respecto.

La presente investigación tiene como finalidad realizar un análisis profundo y exhaustivo de la cobertura de la prensa acerca de los conflictos de Yemen y Ucrania. Dicha investigación se ha efectuado a partir del análisis de los medios de comunicación RT (Rusia), HispanTV (Irán), El Mundo y El País (España), en sus versiones en castellano.

\section{Encuadres, medios y conflictos armados}

Las teorías de encuadres analizan los esquemas de pensamiento en el tratamiento de noticias dentro de ámbitos contextuales específicos y genéricos. $\mathrm{Al}$ igual que en otros temas políticos, el proceso de encuadre en los conflictos bélicos ha creado un corpus de literatura propio, como hemos mencionado anteriormente. El principal marco metodológico sobre el cual se han construido las teorías de encuadres se basa en las características propuestas por Entman (1993: 52): definen problemas, identifican causas, aportan soluciones y establecen juicios morales.

Las teorías de encuadres tienen la finalidad de explicar cómo unos aspectos y consideraciones se destacan sobre otros dentro de un debate político (Bartholomé et al., 2017). Por supuesto, en un discurso no tienen por qué estar presentes las cuatro categorías propuestas por Entman en su totalidad, pero sí al menos una de ellas, lo que a la postre permitirá diferenciar entre encuadres sustantivos y encuadres genéricos. Sin embargo, desde el punto de vista metodológico, la teoría de encuadres sigue siendo considerada un paradigma fragmentado, pues no existe una operacionalización de medidas estándares (Entman, 1993; Al Nahed y Hammond, 2018: 365).

2 Véanse los informes en https://www.rsf-es.org/ 
Estos estudios se han centrado principalmente en cómo los medios de comunicación exponen ante la opinión pública los sucesos políticos (Druckman et al., 2013). El cómo los temas se presentan a la audiencia puede influir en la valoración que esta realiza (priming) y, por tanto, puede modificar los ritmos de la agenda mediática (Neuman, 1992). Por supuesto, los contextos históricos, junto con el proceso de encuadre, influyen, aunque no determinan, las percepciones de la opinión pública.

Los estudios de encuadres y opinión pública han arrojado datos significativos, como el de Blauwkamp et al. (2018: 464-465), donde se muestra que la sociedad norteamericana es favorable al uso de la tortura en el caso del terrorismo, pero la rechaza cuando los medios centran el foco en los métodos de interrogación. Siguiendo esta línea, otros estudios muestran que a raíz de las actividades de ISIS en Indonesia, la prensa ha añadido a los términos islámico o musulmán los conceptos extremistas y radicales, cuando antes no se incidía en la orientación religiosa de los perpetradores de ataques terroristas (Horvit et al., 2018: 460-461).

Los conflictos armados han sido, tradicionalmente, objeto de estudio importante para los analistas de encuadres (García-Marín, 2007). Ello se debe, en primer lugar, a que es fácil ver las relaciones causales entre el origen del encuadre y sus posibles efectos. Son fenómenos fácilmente aislables para su análisis, ya que no intervienen todas las variables de la política nacional (como conocimiento previo, experiencia personal, etc.). En el caso de los conflictos bélicos, y por ende temas asociados con la seguridad, las investigaciones de Semetko y Valkenburg (2000) aportaron cinco encuadres genéricos y comunes a la hora de cubrir contiendas bélicas, que denominaron conflicto, interés humano, consecuencias económicas, moralidad y responsabilidad. Dichas investigaciones mostraron un interés científico extraordinario en cuestiones relacionadas con la seguridad, como el terrorismo, especialmente desde los atentados del 11 de septiembre y el comienzo de la guerra contra el terror (Ette y Joe, 2018: 393).

Por ejemplo, en el caso de uno de los conflictos que nos ocupa, el de Yemen, las rutinas profesionales de los medios hacen que los periodistas (Donsbach y Patterson, 2004) centren sus análisis en los enfrentamientos entre el gobierno yemení y los diferentes actores internos (rebeldes hutíes, grupos leales a Al-Qaeda o Daesh), o entre los distintos protagonistas internacionales (Estados Unidos, Rusia, Irán o Arabia Saudí).

Este análisis puede hacer hincapié en las historias de interés humano de los implicados en la crisis, en las consecuencias económicas internas o externas que produce el conflicto, la moralidad o inmoralidad de las acciones 
militares, o los responsables del conflicto y sus posibles soluciones (Cozma y Kozman, 2018: 189); algo normal si observamos, con base en la literatura, que los encuadres utilizados por la prensa española, francesa y británica son similares y fluctúan dentro de unos marcos explicativos muy concretos (Moreno-Mercado, 2018).

Siguiendo a McDougall (1982), el encuadre conflicto se encuentra más en la cobertura de los medios occidentales, ya que a menudo se emplea para captar el interés de la audiencia (Semetko y Valkenburg, 2000), a través de sucesos impactantes, como las violaciones de derechos humanos o las crisis humanitarias, presentes en cualquier tipo de conflicto armado. Esta afirmación ha sido demostrada actualmente con el empleo de marcos explicativos sobre genocidios y limpiezas étnicas en los conflictos de la Posguerra Fría (Hammond, 2018: 434), llegando incluso a existir dualidad en el propio encuadre entre muertes de mujeres y niños (víctimas) y soldados varones (bajas).

La construcción de encuadres, denominada frame building, engloba uno de los pilares básicos en la programación narrativa de los contendientes. Esto se debe, principalmente, a que enmarcar los conflictos desde enfoques estratégicos concretos permitiría movilizar a las masas populares partidarias, desmovilizar a las contrarias y conseguir apoyo del exterior (Corstange y York, 2018). De ahí, quizá, la importancia de contar con una narrativa propia, traducida en medios como RT e HispanTV, y su rol en los conflictos contemporáneos.

En el caso del canal ruso, para Moscú, el control de los flujos de información y las infraestructuras físicas que lo posibilitan es fundamental, tanto para garantizar la estabilidad doméstica frente a la propaganda extranjera, como para influir en la opinión pública internacional (Colom-Piella, 2017); en especial, porque las vías alternativas de información sirven para crear redes de desinformación exportables a otras plataformas, como Twitter o Whatsapp.

El control de la información y su inserción en la diplomacia pública se han resaltado como lecciones aprendidas esenciales de la caída de la URSS (Colom-Piella, 2018), donde no existía un aparato comunicativo consolidado (más allá de la propaganda), la guerra de Chechenia y el conflicto en Georgia. Lo que explica el uso interesado de encuadres a la hora de cubrir episodios bélicos en los que Rusia se halla inmersa (Moreno-Mercado, 2019), mientras que para el medio persa, propiedad de la Islamic Republic of Iran Broadcasting (IRIB), la finalidad de su programación es contrarrestar las narrativas occidentales, sobre todo en el ámbito de Oriente Medio, e interconectar los flujos de información, en particular en el mundo hispanoamericano, como reconoció el expresidente Mahmud Ahmadineyad (Di Ricco, s.f.: 3). 
Pero, ¿qué elementos empíricos podemos mostrar para diferenciar el rol de un medio de comunicación? ¿Cuándo es propaganda y cuándo información, aunque sea sesgada? El debate es, desde luego, irresoluble desde muchos puntos de vista. Nosotros presentamos aquí una tímida propuesta basada en los encuadres. El origen de la misma es la utilización de las rutinas periodísticas. De hecho, en el corazón de la teoría de encuadres subyace la idea de que muchos de ellos son atajos en la exposición de la información: para informar de una guerra debe haber víctimas, debe haber armas, etcétera. ¿De dónde surge la utilización de rutinas periodísticas? Seguramente de la idea de la competición. Es decir, se repite lo que funciona, porque hay una necesidad de adquirir lectores, clics, televidentes o cualquier otra forma de atención que sea rentable. Es una lógica de mercado que también debería funcionar con los medios públicos, porque ha creado patrones culturales, además de que funcionan con criterios de mercado como las audiencias (Hardy, 2010). Pero, ¿qué pasa cuando el objetivo no es obtener lectores sino promover un determinado punto de vista? Nuestra propuesta es que ahí deberíamos ver alteraciones significativas del uso de estas rutinas que asemejamos (estirando el concepto) a los encuadres.

Llegados a este punto resulta necesario hacer hincapié en que el periodismo de guerra y el periodismo de paz representan dos encuadres competitivos entre sí. El proceso de encuadre conflictivo/bélico se presenta más intensamente en los medios de comunicación y en las redes sociales, tal vez por la mercantilización de la guerra, utilizando con frecuencia encuadres episódicos. La indexación de los medios y la cobertura episódica de los conflictos dificulta la labor de los periodistas para desafiar los problemas estructurales y las justificaciones de los discursos, como muestra DeCillia (2018) al analizar la cobertura por parte de los medios canadienses de la operación militar que se llevó a cabo en Afganistán entre los años 2006 y 2011.

Dichos encuadres episódicos centrados en vencedores y vencidos, basados en enfoques culturales para crear arquetipos concretos, David vs Goliat (Sheafer y Gabay, 2009), hacen hincapié en las bajas civiles y los sucesos violentos. Un ejemplo claro lo encontramos en la investigación de Wolfsfeld (2018: 120), donde muestra cómo las redes sociales, en referencia al conflicto palestino-israelí, son mucho más propensas a difundir contenido violento que a fomentar mecanismos de resolución y paz.

Por el contrario, el periodismo de paz centra su cobertura en el uso de encuadres genéricos, basados en las responsabilidades del enfrentamiento bélico marcados por las raíces históricas del conflicto (Galtung, 2007). En este sentido, el papel activo de los medios en la comunicación y gestión constructiva 
de crisis es conocida entre los programas de investigación como periodismo de paz, periodismo positivo y periodismo constructivo (Lichtenstein et al., 2018: 4). Sin embargo, recientes aportaciones científicas siguen señalando que los hechos violentos, y en especial las graves consecuencias de los conflictos armados nacionales e internacionales, continúan ocupando la mayoría de las noticias y editoriales de la prensa escrita, televisión y radio (Gouse, et al., 2018: 10).

\section{Los conflictos de Yemen y Ucrania, una breve introducción}

El primer caso de estudio es el conflicto que actualmente asola la República de Yemen (desde 2015). Las revueltas del año 2011, también conocidas como Primavera Árabe o Despertar Árabe, afectaron al país; el presidente Ali Abdullah Saleh abandonó el poder tras más de 30 años en el cargo, siendo Yemen un ejemplo de transición pacífica del poder, en su etapa inicial (Medina-Gutiérrez, 2018: 95). La intervención de la coalición liderada por Arabia Saudí contra las fuerzas hutíes, ${ }^{3}$ respaldadas por el régimen de Teherán que tomaron Saná en 2014, ha dejado más de 6.782 víctimas mortales (United Nations and Word Bank, 2018). El conflicto yemení ha tenido un antes y un después desde el golpe de Estado contra el presidente interino Abd Mansur al-Hadi por parte de la insurgencia hutí, lo cual ha propiciado una sangrienta guerra civil con dos gobiernos enfrentados, en lo que algunos académicos han denominado Guerra Fría Iranio-Saudí (Salisbury, 2015; Tzemprin et al., 2015).

Por supuesto, no solo el conflicto armado entre las partes ha atraído la atención mediática internacional en Yemen. Por un lado, la emergencia del ISIS en la región MENA ha transformado el paradigma de seguridad y las relaciones internacionales en la zona (Durán y Bados, 2017: 294), incluido el país árabe. Y, por otro, las implicaciones de las grandes potencias regionales ha provocado drásticas consecuencias humanitarias para el país, como el bloqueo naval ejercido por la marina saudí, ${ }^{4}$ y tensiones diplomáticas con otras grandes potencias, como las tirantes relaciones de Irán con el presidente estadounidense Donald Trump.

El segundo caso de estudio aquí planteado, el conflicto de Ucrania o también denominado guerra en el Donbáss (iniciado en 2014), presenta otras implicaciones. El eje ucraniano, más importante para Rusia que otras áreas

3 También conocidos como Ansar Allah o Partisanos de Dios.

4 Donde podemos destacar la polémica producida en Europa sobre la venta de armas a la Casa de los Saúd, llegando incluso Alemania a cancelar la venta de armamento. 
limítrofes, como Georgia según algunos expertos (Tsygankov, 2015: 281), ha dejado, según las estimaciones de las Naciones Unidas, más de 10.300 muertos (Colás, 2015). Las protestas del Euromaidán, que dieron lugar al auge de sectores europeístas contrarios a Rusia, son vistas no solo como una limitación regional por el Kremlin, sino como un proyecto por parte de la OTAN de reconfigurar el sistema político ruso a mediano plazo (Hove, 2016: 151).

El conflicto en Ucrania es considerado la crisis europea más grave tras el fin de la existencia del bloque soviético (Burns, 2015: 63), después de las guerras balcánicas. El conflicto armado ha ocupado la atención mediática en la prensa europea, no solo por las implicaciones de dicha guerra, asociadas al uso de gaseoductos provenientes de Rusia, sino por la aparición de grupos paramilitares nacionalistas en amplias zonas del país como Pravy Sektor o milicias asociadas al partido extremista Svoboda. ${ }^{5}$

En el caso del país europeo, autores como Baqués (2017) o Jordán Enamorado (2018b: 130) defienden que no nos encontramos ante una Nueva Guerra Fría, sino ante "conflictos en la zona gris", especialmente circunscritos en el enfoque del realismo ofensivo de Mearsheimer (2003). Dentro de este concepto se integra una serie de elementos definitorios, como la ambigüedad de relaciones ni pacíficas ni de conflicto armado, estrategias multidimensionales, intereses sustanciales en juego y gradualismo (Jordán Enamorado, 2018a: 131-133). Por otra parte, autores como Steele (2015) o Legvold (2018) rechazan el término, debido a la inexistencia de dos bloques globales e ideológicamente contrapuestos, como sí se dieron entre 1947 y 1991.

\section{Metodología}

Como se puede apreciar, ambos conflictos resultan adecuados para intentar cumplir el objetivo de la presente investigación: conocer si las rutinas periodísticas de nuevos medios como RT e HispanTV se asemejan a las rutinas de los medios tradicionales cuando cubren sucesos internacionales. Nuestro trabajo tiene cuatro objetivos específicos:

- Analizar, a partir de las teorías de encuadres, grandes volúmenes editoriales de los medios seleccionados cuando cubren los conflictos de Yemen y Ucrania.

- Conocer si existen diferencias notables en el uso de los encuadres drama humanitario y seguridad.

5 En castellano, Sector Derecho y Libertad, respectivamente. 
- Determinar si podemos considerar teóricamente que RT e HispanTV siguen las rutinas profesionales de los medios de comunicación a la hora de cubrir conflictos bélicos.

La hipótesis de partida es la siguiente:

H1: El uso de encuadres por parte de RT e HispanTV diferirá significativamente en los dos conflictos analizados.

Para la demostración de la hipótesis, se ha decidido explorar la cobertura mediática de los conflictos de Yemen y Ucrania en dos medios tradicionales y dos medios alternativos. Es decir, utilizaremos la cobertura de dos medios tradicionales en castellano para compararla con el análisis de los dos medios objetivo de esta investigación.

En concreto, el estudio de medios de comunicación está integrado por las versiones digitales de El País, El Mundo (España), RT (Rusia) e HispanTV (Irán), en sus formatos en castellano. Las piezas editoriales han sido extraídas de la base de datos de Lexis-Nexis Academic, muy útil para recolectar grandes cantidades de textos periodísticos y los monitores de búsqueda propios de $R T$ e HispanTV. Las cadenas de búsqueda tuvieron los siguientes términos, guerra*, Yemen*, Ucrania*, Donbass*, conflicto*, hutíes*, prorusos*, siendo el asterisco $\left(^{*}\right)$ un parámetro que indica la inclusión de palabras derivadas.

El periodo de estudio comprende los últimos cuatro años de ambos conflictos (enero de 2015-febrero de 2019), periodo altamente significativo por la existencia de sucesos de gran impacto mediático en ambos conflictos, como el ataque de la coalición saudí al puerto de Hodeida (Yemen) o las tensiones entre la Federación Rusa y la República de Ucrania, por las consecuencias tras las adhesiones de Crimea y Sebastopol. No obstante, cabe señalar que la muestra presenta algunas limitaciones. En el caso de $R T$ no se ha podido analizar el año 2015, debido a que ha sido imposible extraer dichos datos al no ser accesibles en su motor de búsqueda.

El resultado final de búsqueda y extracción, y su posterior limpieza, fue de 4.323 piezas editoriales, lo cual demuestra el amplio alcance de la investigación. Los datos fueron tratados con el software estadístico $R$ para aplicar el algoritmo supervisado SVM (Support Vector Machines). El uso de dicho tipo de algoritmos resulta ideal para estudios como este (Joachims, 1998). Principalmente, porque se ha demostrado que el SVM es más efectivo que las redes neuronales (Osowski et al., 2004) en tareas de clasificación (no regresión), siendo perfecto para textos de larga extensión, como las noticias, utilizando diferentes kernerls. Además, como señalan García-Marín et al. (2018: 627), "desde nuestra experiencia es más fiable que los codificadores humanos si están bien entrenados”. 
El uso de Máquinas de Soporte Vectorial (SVM) no es una herramienta metodológica de incipiente utilización en computación (Cortés y Vapnik, 1995), pero sí en campos como la comunicación política, limitando su presencia a unos pocos estudios (García-Marín y Calatrava-García, 2018; García-Marín et al,. 2018). La característica común de este algoritmo es su escasa complejidad. Como señala Gala-García (2013: 6), "es una de las técnicas más poderosas de aprendizaje automático que, a pesar de su sencillez, ha demostrado ser un algoritmo robusto y que generaliza bien en problemas de la vida real".

A pesar de que el origen de las SVM se encuentra en la resolución de clasificaciones binarias, su uso se ha extendido con éxito a otros campos y problemas, como regresiones, categorización de texto e hipertexto y procesamiento de lenguaje natural, entre otros (Carmona-Suárez, 2014). No obstante, la finalidad de la técnica es la misma, ocupar un hiperplano, o separador lineal en un espacio de alta multidimensionalidad que separe las categorías utilizadas. En palabras de Carmona-Suárez (2014: 2): "la búsqueda del hiperplano de separación en estos espacios transformados, normalmente de muy alta dimensión, se hará de forma implícita utilizando las denominadas funciones kernel'.

En el caso de que los datos usados no sean linealmente separables, podemos ocupar una serie de herramientas kernel, como polinomio, sigmoidal o gaussiana entre otras. La solución del hiperplano óptimo puede ser descrita "como la combinación de unos pocos puntos de entrada que son llamados vectores de soporte" (Betancourt, 2005). Como indica la literatura computacional, el método de aprendizaje de SVM está más encaminado a minimizar el riesgo estructural, lo cual favorece estructuras simples, que el riesgo empírico producido por errores de aprendizaje del modelo generado a partir de su entrenamiento. Esta función resulta altamente positiva, debido a que la solo minimización del riesgo empírico puede producir un sobreajuste.

Para poder trabajar con textos largos (minería de textos o procesamiento de lenguaje natural), como en el caso de las noticias, resulta especialmente significativo el peso y la frecuencia de palabras que lo componen $(t f)$. No obstante, en estos textos existe una gran cantidad de palabras que pueden alterar el análisis y que en realidad no son relevantes, como las preposiciones. De ahí la necesidad de utilizar las librerías disponibles en $R$, como el caso de tm para eliminar palabras centrales altamente repetidas, eliminar la puntuación, uniformar en minúsculas el texto, eliminar los espacios en blanco y suprimir las stopwords, para conseguir textos tokenizados. 
Igualmente, para determinar el nivel de presencia de temáticas dentro de los textos analizados, resulta óptimo trabajar con el cálculo de factor $t f-i d f$. El método $t f$-idf genera listas de palabras clave con una calificación o peso que indica cuán relevante es la palabra respecto al documento seleccionado y al corpus en general (Cabrera, 2011). A pesar de que este método ha presentado limitaciones (Ramos, 2003), al no detectar sinónimos y plurales, los errores de producción son fácilmente subsanables mediante la utilización de procesos de stemming. Estos consisten en el proceso de eliminación automática "de partes no esenciales de los términos (sufijos y prefijos) para reducirlos a su parte esencial (lema) y ayudar a la correcta indización y recuperación" (Vuotto et al., 2015: 3).

La implementación usada en esta investigación fue "libsvm" (Chang y Ling, 2011), a través de "e1071" (Grün y Hornik, 2011; Meyer, 2001), y la librería para transformar los datos en una matriz inversa "tm" (Feinerer et al., 2008), como se ha mencionado. Dichas librerías se encuentran en código abierto y son consideradas como estándares en la utilización de SVM. A través de "tm" se transformaron los textos en tokens.

El entrenamiento del algoritmo se realizó con la codificación de 600 editoriales de manera aleatoria (random). Dicha codificación se efectuó de forma dicotómica (presencia/ausencia) de los encuadres por analizar codificados a partir de la definición del problema. Principalmente, porque los sucesos internacionales suelen ser cubiertos con base en esta característica propuesta por Entman (1993). No se realizó comprobación de su fiabilidad, pues la creación de un modelo válido ya es prueba más que suficiente de su viabilidad (García-Marín et al., 2018). Empero, el propio modelo realiza una predicción de su propia viabilidad, sirviendo también para limpiar la base datos localizando unidades de análisis que no debía clasificar (asignando 0,50 de probabilidad).

Finalmente, se probó el algoritmo con 121 unidades aleatorias más, dando un resultado de $86 \%$ de fiabilidad entre la clasificación realizada por los codificadores y el algoritmo, lo cual, aunque hubiera sido deseable que fuera más alta, avala la viabilidad de la investigación.

\section{Resultados}

El análisis descriptivo de los datos nos muestra las diferencias presentes en la cobertura de los medios estudiados. Como muestra la Tabla $1,{ }^{6}$ el medio iraní HispanTV es el que dedica mayor cobertura a ambos conflictos con 6 La tabla y los gráficos se encuentran en el Anexo, al final del presente artículo (Nota del editor). 
diferencias muy notables respecto a los otros, con 622 editoriales (45.87\%) dedicadas a la crisis ucraniana y $2.154(74.37 \%)$ en el caso de Yemen. Si bien es cierto, como se comentó anteriormente, que no se han podido extraer las editoriales de $R T$ del año 2015, las diferencias respecto a HispanTV son tan significativas que probablemente no hubieran alterado de forma significativa los datos expuestos. $R T$ queda como el segundo medio que más editoriales dedica a los conflictos con 300 editoriales para la crisis ucraniana $(22,12 \%)$ y 292 para el caso de Yemen (10,08\%). Mientras que los diarios españoles El Mundo y El País quedan relegados a una modesta representación del total de la muestra analizada, no llegando en ninguno de los dos casos a más del 30\%. Esto era realmente lo esperado, ya que los diarios españoles tienen formato físico, a diferencia de los otros medios, y su grado de interés en los conflictos resulta menor por la lejanía de los mismos (véase Tabla 1 ).

El carácter internacional de $R T$ e HispanTV-puesto que ambos admiten tener un importante objetivo en América Latina-, puede explicar también las diferencias cuantitativas referentes al número de editoriales. No obstante, resulta significativo que el medio iraní dedique un 23,75\% más de cobertura que el canal ruso. La explicación de esto podemos encontrarla en que el tamaño medio de las noticias de HispanTV es más reducido, pero a la vez más abundante, que en las del medio ruso. Las frecuencias extraídas refuerzan la escasa literatura existente sobre $R T$. Moreno-Mercado (2019: 111) señala al respecto: "La posición rusa en el este de Ucrania ha ido evolucionando desde la omisión directa de implicaciones y el uso de fuerzas regulares de su ejército a la detención de marinos ucranianos en el estrecho de Kerst en noviembre de 2018".

Durante la crisis en el Donbass, el Ejecutivo ruso ha negado reiteradamente su presencia militar en el país europeo, llegando el presidente Vladimir Putin a declarar "En el territorio del Donbass no hay tropas rusas. Aunque es verdad que allí se han creado unidades militares con milicianos que son autosuficientes" (La Vanguardia, 2017). A pesar de las múltiples contradicciones informativas, los datos muestran la tendencia del medio ruso sobre qué temas obviar y en cuáles incidir, ya que la cobertura sobre el conflicto en el país árabe - geográfica y estratégicamente mucho menos determinante- resulta muy similar. En este sentido, cobra gran importancia la frase de Bernard Cohen (1991: 9): "Si el gobierno llegara a perder su poder para gestionar la cobertura de las noticias en un escenario de guerra, su capacidad para usar la fuerza militar puede perderse".

Podría decirse que el potencial mediático de la guerra yemení posee una doble dimensión. Por un lado, la ONU ha arrojado cifras alarmantes de la situación en el país árabe, asegurando que $70 \%$ de la población se encuentra en 
riesgo de inseguridad alimentaria (United Nations and Word Bank, 2018). Por otro lado, el conflicto ha sido catalogado como un rescoldo más del enfrentamiento entre las grandes potencias regionales de la región, Arabia Saudí e Irán.

El Gráfico 1 señala las fluctuaciones mediáticas que ha sufrido el conflicto. Una conclusión rápida que podemos extraer es la coincidencia de aumento de cobertura en HispanTV sobre las acciones bélicas desarrolladas por la coalición saudí. El momento de mayor cobertura del medio iraní coincide con el golpe de Estado perpetrado por las milicias hutíes a principios del año 2015, que iniciaron la intervención militar del Consejo de Cooperación para los Estados Árabes del Golfo (CCEAG).

Como se puede inferir, la cobertura del medio persa ha sido constante durante el desarrollo de las hostilidades, produciéndose picos significativos cuando la coalición árabe ha realizado ofensivas de gran magnitud, resultados que se encuentran en la línea de lo esperado. Prueba de ello son los más de 17 civiles muertos en la provincia de Saada, con titulares como "Bombardeos de Arabia Saudí dejan 10 civiles muertos en Yemen" (HispanTV, 2017), o los ataques al estratégico puerto de Hodeida a pesar del alto el fuego pactado con la ONU, con titulares como "Ataque saudí contra sede de seguridad deja 60 muertos en Yemen”(HispanTV, 2018) (véase Gráfico 1).

Por otro lado, la cobertura de los otros tres diarios ha sido fundamentalmente homogénea, produciéndose alteraciones en momentos relevantes del conflicto, como las sanciones norteamericanas a Irán por el armamento de las milicias hutíes, con noticias como: "Pompeo aterriza en Arabia Saudita e inmediatamente pide nuevas sanciones contra Irán” ( $R T$, 2017) o circunstancias políticas internas en el caso español. En el Gráfico 1 se puede ver cómo la cobertura en los medios españoles aumenta en medio del debate sobre la venta de armas del gobierno español a Arabia Saudí y los contratos de la monarquía árabe con la empresa española Navantia, situada en Cádiz, con noticias como la de El Mundo: "Moncloa vuelve a frenar la venta de armamento a Arabia Saudi" (Segovia, 2019) o la de El Pais: "Barcos sin principios" (Yárnoz, 2018).

En el caso ucraniano se dan matices importantes. El Gráfico 2 muestra cómo la cobertura de $R T$ decrece a medida que el conflicto aumenta su dimensión armada. Podemos encontrar la explicación en las reiteradas negaciones del Kremlin sobre la presencia de militares rusos en la antigua república soviética y los principios estratégicos informativos rusos sobre qué temas obviar para lograr mayor cuota de poder blando (Sánchez-de Rojas Díaz, 2018); especialmente porque el público potencial de $R T$ son ciudadanos 
europeos que buscan un punto de vista alternativo a las narrativas de los medios occidentales. Al igual que en el caso yemení, la cobertura presenta fluctuaciones, aunque estas son menores y menos constantes.

Si en la guerra civil yemení el volumen de noticias se encuentra ligado a acciones u ofensivas militares y crisis humanitarias, el conflicto ucraniano se encuentra marcado por las crisis diplomáticas entre Rusia, la Unión Europea y Estados Unidos. Ejemplos ilustrativos son las noticias referentes al derribo del avión malayo en territorio ucraniano: "La UE y la OTAN piden cuentas a Rusia por el derribo del avión malasio" (Abellán, 2018) de El Pais, o cuestiones geopolíticas, como "Assad, Putin y Jamenei pueden dormir tranquilos” (Sahagún, 2018) de El Mundo. Por el contrario, la cobertura rusa e iraní incide más en las características del conflicto, sin olvidar la dimensión geopolítica: "Lo último de la escalada del conflicto en el este de Ucrania" (RT, 2016) (véase Gráfico 2).

En cuanto al uso de encuadres y su peso, el Gráfico 3 muestra que el encuadre seguridad posee un mayor peso que drama humanitario en los cuatro medios analizados en referencia al caso ucraniano. Puede decirse que los datos coinciden con las expectativas esperadas. Las últimas investigaciones sobre medios y conflictos señalan cómo en los medios europeos el encuadre de seguridad posee un mayor peso que el humanitario, siendo el caso sirio una buena lección de ello (Moreno-Mercado, 2018). A pesar de que el componente humanitario posee escasas diferencias con el de seguridad, puede decirse que los medios españoles aportan una visión europeísta y atlantista de la crisis, como esta nota de El País: "La OTAN se refuerza ante las crecientes amenazas en su flanco sur” (González, 2015).

Asimismo, $R T$ encuadra el conflicto a partir de la amenaza europea a la soberanía rusa y la persecución de población rusófona en Ucrania: "La flota rusa del mar Negro inicia ejercicios de operaciones anti sabotaje” (RT, 2017); algo lógico, ya que las variables geográficas influyen en gran medida, que no determinan, el comportamiento exterior de los Estados (Jordán Enamorado, 2018a). Igualmente, HispanTV centra su información en las implicaciones estadounidenses en la desestabilización de la región: "EEUU destina 350 millones de dólares en ayuda militar a Ucrania” (HispanTV, 2015) (véase Gráfico 3).

En cuanto al uso de encuadres en la crisis yemení, los resultados son radicalmente opuestos. El encuadre humanitario es el que presenta más significatividad en los cuatro medios. Los motivos en el caso español pueden traducirse en varias explicaciones. Por un lado, la guerra en Yemen no está produciendo oleadas de refugiados a Europa, lo cual implica que no existan, 
ni en el ámbito político ni mediático, narrativas que hagan hincapié en la seguridad, lo que sí ocurre en otros casos, como los de Siria o Libia (Rettberg y Gajjala, 2016).

En tanto, HispanTV utiliza mayoritariamente el encuadre bumanitario centrándolo en el rol de Arabia Saudí, por lo que sería interesante ver en futuras investigaciones el peso de atribución de responsabilidades, ya que las alusiones a la culpabilidad de la Casa de Saúd en la muerte de civiles yemeníes son constantes. Finalmente, el medio ruso $R T$ también recurre a este encuadre, aunque podríamos considerar su uso como una línea mediática coyuntural, por su papel en el conflicto sirio y el juego de alianzas que se desarrollan en la antigua colonia francesa: " $¿$ Ayuda Trump a Arabia Saudita a destruir Yemen?” (RT, 2018) (véase Gráfico 4).

El uso de encuadres y su distribución arrojan datos muy clarificadores (véase Gráfico 5). Por un lado, la cobertura de los medios ruso e iraní supera con creces el espacio informativo que los medios tradicionales españoles dedican a estos conflictos armados; lo que hace suponer el interés que tiene para ambas redacciones.

Finalmente, cabe destacar que si estudios como los de Pantti (2016) afirmaban que los medios de países democráticos utilizaban leyendas humanitarias en sus noticias en contraposición a los medios de países no democráticos, en este caso no se cumple dicha afirmación, pues el uso de explicaciones más humanitarias se encuentra íntimamente relacionado con los intereses geoestratégicos de los países en cuestión; ya que las diferencias en la utilización de encuadres en los medios españoles no supera el $10 \%$, mientras que en HispanTV la diferencia se sitúa en 30\% (30/70\% en Ucrania y $60 / 40 \%$ en Yemen) y en $R T$ se dan los datos más significativos con $90 \%$ (90/10\% en Ucrania y 40/60\% en Yemen). Estos resultados confirman la hipótesis planteada anteriormente. Como se puede observar, el comportamiento de los medios ruso e iraní no presenta procederes uniformes, lo cual hace que podamos intuir que sus rutinas profesionales no se equiparan a las de otros medios clásicos, coincidiendo el uso de encuadres con sus intereses geoestratégicos.

\section{Conclusiones}

Este trabajo muestra cómo es el tratamiento que los medios tradicionales españoles y los medios alternativos, RT e HispanTV, realizan acerca de los conflictos de Yemen y Ucrania. 
Al comienzo de nuestro trabajo planteábamos la hipótesis de que el uso de encuadres en $R T$ e HispanTV dependería de los intereses geoestratégicos que sus países de origen mantienen en los conflictos analizados. Como muestra el presente estudio, nuestra hipótesis ha quedado validada, señalando diferencias significativas con los medios tradicionales españoles.

Se ha demostrado que estas divergencias son especialmente significativas en el caso de $R T$, donde el peso de los encuadres se encuentra íntimamente relacionado con el comportamiento exterior que está desarrollando la Federación Rusa en los últimos años, lo cual refuerza empíricamente lo que ya hacían otros estudios (Yablokov, 2015; Keating y Kaczmarska, 2019). Por otro lado, aunque en el medio iraní los datos arrojan resultados menos evidentes, las diferencias mediáticas siguen siendo muy significativas.

Nuestra interpretación de los datos obtenidos está relacionada con las rutinas profesionales de los medios. En nuestra opinión, las diferencias mediáticas radican no en que estos medios sean de propiedad estatal, sino en que sus rutinas mediáticas no siguen las pautas de los medios tradicionales, que hemos representado, en este caso, con los españoles.

Lo anterior nos lleva a inferir que teóricamente no podemos catalogar a estos medios como tradicionales o, al menos, como medios homologables a lo que encontramos, mayoritariamente, en los países democráticos. También puede aducirse que las diferencias pueden deberse a entornos culturales diferentes o a contextualizaciones de otro tipo. Sin embargo, el principal hallazgo no radica en las diferencias de cobertura respecto a los medios españoles (que se han usado básicamente como grupo de control), sino entre sí mismos. Es decir, ¿por qué se dan diferencias tan significativas entre los propios medios analizados?

El caso de $R T$ es paradigmático: un $90 \%$ de diferencia en el uso de encuadres cuando se informa de conflictos que, en esencia (en lo trágico), no son tan diferentes. HispanTV se comporta de forma similar, aunque menos extrema. Pero los dos casos comparten presencias y, sobre todo, ausencias importantes, lo cual puede ayudar a establecer la clave de la discusión sobre los motivos e implicaciones de los conflictos: humanitario en el caso de Yemen, geopolítico en el caso ucraniano.

Los datos sugieren que hay una motivación por ocupar el espacio informativo quizá para imponer agendas políticas concretas (recordemos que son medios en castellano, no dirigidos a sus públicos nacionales), probablemente porque en los sucesos propios de la política exterior se dan las condiciones idóneas para la manipulación informativa (Jacobs, 2001). 
No obstante, somos conscientes de las limitaciones que este estudio presenta, debido a que los resultados tienen un carácter exploratorio. Sería interesante y recomendable investigar el comportamiento de otros medios europeos de países democráticos, como los casos de diarios franceses, británicos y alemanes, además de otros medios de propiedad pública, como Radio Televisión Española (RTVE). Igualmente, ampliar los casos de estudio. Siria, por su repercusión mediática y geopolítica, sería ideal para completar lo iniciado en esta investigación.

Sería interesante ahondar en el uso y efecto de fake news en próximos trabajos. Resulta importante destacar el valor de esta investigación, ya que puede servir de base para los futuros estudios de conflictos, medios de comunicación, paz (en especial a partir del peace journalism), seguridad y defensa que deseen aproximarse a la dimensión informativa de las guerras; sobre todo, por su carácter exhaustivo y completo al contar con una muestra de más de 4.323 unidades de análisis, lo que, a nuestro juicio, es una contribución novedosa que abarca más allá de la comunicación política.

Cabe destacar la importancia y éxito de la metodología utilizada. Principalmente, porque es la primera vez que ha sido empleada para examinar la relación entre medios de comunicación y conflictos armados, aunque ya ha sido aplicada satisfactoriamente en otros estudios. Esta investigación, pues, permite reforzar el uso de algoritmos supervisados en estudios de comunicación política, además de permitir confirmar y refutar hipótesis de trabajo más ambiciosas por las capacidades que estas técnicas de análisis admiten.

\section{Referencias}

Abellán, Lucía (2018), "La UE y la OTAN piden cuentas a Rusia por el derribo del avión malasio", en El País, España.

Al-Nahed, Sumaya y Hammond, Philip (2018), "Framing War and Conflict: Introduction to the Special Issue", en Media, War \& Conflict, vol. 11, núm. 4, Estados Unidos: Sage Journals.

Bartholomé, Guus et al. (2017), "Towards a Tipology of Conflict Frames: Substantiveness and intervetionsm in political conflict news", en Journalism Studies, vol. 19, núm. 12, Reino Unido: Taylor and Francis.

Baqués, Josep (2017), "El papel de Rusia en el conflicto de Ucrania: ¿La guerra híbrida de las grandes potencias?", en Revista de Estudios en Seguridad Internacional, vol. 1, núm. 1, España: Grupo de Estudios en Seguridad Internacional.

Betancourt, Gustavo (2005), "Las máquinas de soporte vectorial (SVMs)", en Scientia et technica, vol. 1, núm. 27, Colombia: Universidad Tecnológica de Pereira.

Blauwkamp, Joan et al. (2018), "Are Americans really okay with torture? The effects of message framing on public opinion", en Media, War \& Conflict, vol. 11, núm. 4, Estados Unidos: Sage Journals. 
Convergencia Revista de Ciencias Sociales, vol. 27, 2020, Universidad Autónoma del Estado de México

Burns, Nicholas (2015), “The Aspen Strategy Group on the Old and New Cold War”, en The Aspen Idea, Estados Unidos: The Aspen Idea.

Cabrera, Diego (2011), TF-IDF para la obtención automática de términos y su validación mediante Wikipedia, México: Universidad Nacional Autónoma de México.

Canel, María José (1999), Comunicación politica. Técnicas y estrategias para la sociedad de la información, España: Tecnos.

Carmona-Suárez, Enrique (2014), Tutorial sobre máquinas de vectores de soporte (SVM), España: Universidad Nacional de Educación a Distancia (UNED).

Chang, Chih-Chung y Ling, Chih-Jen (2011), "Libsvm: A library for support vector machines”, en ACM transactions on intelligent systems and technology (TIST), vol. 2, núm. 3, China: ACM Digital Library.

Cohen, Bernard (1991), "A View from the Academy”, en Bennett, Lance y Paletz, David [eds.], Taking by Storm: The Media Public Opinion and US Foreign Policy in the Gulf War, Estados Unidos: Chicago University Press.

Colás Xavier (2015), "Putin anuncia un alto el fuego en el este de Ucrania este domingo", en El Mundo. Disponible en: https://www.elmundo.es/internacional/2015/02/12/54dc 561a268e3ebe438b456e.html [3 de mayo de 2019].

Colom-Piella, Guillem (2017),"Rusia y las operaciones de información”, en Blog Mosaico, España: Grupo de Estudios en Seguridad Internacional.

Colom-Piella, Guillem (2018) "La doctrina Gerasimov y el pensamiento estratégico ruso contemporáneo”, en Revista Ejército, núm. 933, España: Ministerio de Defensa.

Corstange, Daniel y York, Erin (2018), "Sectarian framing in the Syrian Civil War", en American Journal of Political Science, vol. 62, núm. 2, Estados Unidos: Midwest Political Science Association.

Cortés, Corinna y Vapnik, Vladimir (1995), "Support-Vector Networks", en Machine Learning, vol. 20, núm. 3, Alemania: Springer.

Cozma, Raluca y Kozman, Claudia (2018), "The Syrian crisis in US and Lebanese newspapers: A cross-national analysis", en International Communication Gazzette, vol. 80, núm. 2, Estados Unidos: Sage Journals.

DeCillia, Brooks (2018), “But it is not getting any safer”: The contested dynamic of framing Canada's military mission in Afghanistan”, en Canadian Journal of Political Sciencel Revue Cannadiene de Science Politique, vol. 51, núm. 1, Reino Unido: Cambridge University Press.

Di Ricco, Massimo (s.f.), "No News from Tehran: HispanTV First Voyage to Latin America", en Working Paper. Programa Medio Oriente, núm. 31, Argentina: Centro Argentino de Estudios Internacionales.

Donsbach, Wolfgang y Patterson, Thomas (2004), "Political News Journalists", en Esser, Frank y Pfetsch, Barbara [eds.], Comparing Political Communication: Theories, cases and challenges, Reino Unido: Cambridge University Press.

Druckman, James et al. (2013), "How elite partisan polarization affects public opinion formation”, en American Political Science Review, vol. 107, núm. 1, Reino Unido: Cambridge University Press.

Durán, Marién y Bados, Víctor (2017), “The Political and Security Repercussions of the Islamic State in the Middle East and North Africa Region”, en Szmolka Vida, Inmaculada [ed.], Political Change in the Middle East and North Africa: After the Arab Spring, Reino Unido: Edinburgh University Press. 
Entman, Robert (1993), “Framing: Toward clarification of a fractured paradigm”, en Journal of Communication, vol. 43, núm. 4, Estados Unidos: International Communication Association.

Ette, Mercy y Joe, Sarah (2018), “Rival visions of reality”: An analysis of the framing of Boko Haram in Nigerian newspapers and Twitter”, en Media, War \& Conflict, vol. 11, núm. 4, Estados Unidos: Sage Journals.

Feinerer, Ingo et al. (2008), “Text mining infraestructure in R”, en Journal of Statistical Sowftware, vol. 25, núm. 5, Estados Unidos: Foundation for Open Access Statistics.

Gala-García, Yvonne (2013), Algoritmos SVM para problemas sobre big data, España: Universidad Autónoma de Madrid.

Galtung, Johan (2007), “Peace Journalism as Ethical Challenge”, en Asteriskos, vol. 3, núm. 4, España: Galician Institute for International Security and Peace Studies.

García-Marín, Javier (2007), El comportamiento de la prensa durante los conflictos de Kosovo e Irak, España: Universidad de Granada.

García-Marín, Javier y Calatrava-García, Adolfo (2018), “The Use of Supervised Learning Algorithms in Political Communication and Media Studies: Locating Frames in the Press", en Communication \& Society, vol. 31, núm. 3, España: Universidad de Navarra.

García-Marín, Javier et al. (2018), "Debates electorales y conflicto: Un análisis con máquinas de soporte virtual (SVM) de la cobertura mediática de los debates electorales en España desde 2008”, en El Profesional de la Información, vol. 27, núm. 3, España: El Profesional de la Información.

García-Marín, Javier y García-Luengo, Óscar (2009), "Politics, Media and War Coverage: An Indexed Relation?”, en Van Vaarda, Ted y Verweij, Desiré [eds.], The Moral Dimension of Asymetrical Warfare, Países Bajos: Martinus Nijhoff.

Gerasimov, Valerie (2013), "El valor de la ciencia en la anticipación”, en ВоенноПромышленный Куреьр, vol. 8, Rusia: Ministerio de Defensa.

González, Miguel (2015), "La OTAN se refuerza ante las crecientes amenazas en su flanco sur”, en El País, España.

Gouse, Valerie et al. (2018), "An investigation of the conceptualization of peace and war in peace journalism studies of media coverage of national and international conflicts", en Media, War \& Conflict, Estados Unidos: Sage Journals.

Grün, Bettina y Hornik, Kurt (2011), “Topicmodels: An R package for fitting topic models”, en Journal of Statical Sowftware, vol. 40, núm. 13, Estados Unidos: Foundation for Open Access Statistics.

Hammond, Philip (2018), "When frames collide: 'Ethnic war' and 'genocide”, en Media, War \& Conflict, vol. 11, núm. 4, Estados Unidos: Sage Journals.

Hardy, Jonathan (2010), Western Media Systems, Inglaterra: Routledge.

Hiller, Debbie y Cairns, Edmund (2018), “The Figthting Must Stop-To Create a Space for Peace: Attacking Hodeida city, after its airport, would be a far greater threat to Yemeni civilians, en Oxfam Library, Reino Unido: Oxfam Repository.

HispanTV (2015), "EEUU destina 350 millones de dólares en ayuda militar a Ucrania”, en HispanTV, Irán.

HispanTV (2017), “Bombardeos de Arabia Saudí dejan 10 civiles muertos en Yemen”, en HispanTV, Irán.

HispanTV (2018), "Ataque saudí contra sede de seguridad deja 60 muertos en Yemen" en HispanTV, Irán. 
Convergencia Revista de Ciencias Sociales, vol. 27, 2020, Universidad Autónoma del Estado de México

Hove, Mediel (2016), “The Emergence of the New Cold War: The Syrian and Ukraine Conflicts”, en Jadavpur Journal of International Relations, vol. 20, núm. 2, Estados Unidos: Sage Journals.

Horvit, Beverly et al. (2018), “23. Journalism, War and Peace”, en Vos, Tim [ed.], Journalism, Alemania: De Gruyter.

Joachims, Thorsten (1998), “Text categorization with support vector machines: Learnign with many relevant features”, en Nédellec, Claire y Rouveirol, Céline [eds.], Machine Learning: ECML-98. ECML 1998. Lecture notes in computer science, Alemania: Springer.

Jacobs, Lawrence (2001), "Manipulators and manipulation: Public Opinion in a representative democracy", en Journal of Health Politics, Policy and Law, vol. 26, núm. 6, Estados Unidos: Duke University Press.

Jordán-Enamorado, Javier (2018a), "Un modelo de análisis geopolítico para el estudio de las relaciones internacionales”, en Bie3: Instituto Español de Estudios Estratégicos, núm. 9, España: Ministerio de Defensa.

Jordán-Enamorado, Javier (2018b), "El conflicto internacional en la zona gris: una propuesta teórica desde la perspectiva del realismo ofensivo", en Revista Española de Ciencia Politica, vol. 48, España: Asociación Española de Ciencia Política.

Keating, Vicent y Kaczmarska, Katarxyna (2019), "Consevative soft power: liberal soft power bias and the 'hidden' attraction of Russia”, en Journal of International Relations and Development, vol. 22, núm. 1, Reino Unido: Palgrave Macmillan.

La Vanguardia (2017), "Putin niega que en Ucrania haya tropas rusas", en La Vanguardia, España.

Legvold, Robert (2018), "Managing the New Cold War: What Moscow and Washington can learn from last one”, en Foreing Affairs, vol. 93, núm. 4, Estados Unidos: Council on Foreign Relations.

Lichtenstein, Dennis et al. (2018), "Framing the Ukraine crisis: A comparison between talk show debates in Russian and German television", en International Communication Gazzette, vol. 81, núm. 1, Estados Unidos: Sage Journals.

MacDonald, Paul (2018), “America First? Explaining Continuity and Change in Trump's Foreign Policy”, en Political Science Quarterly, vol. 133, núm. 3, Estados Unidos: Academy of Political Science.

McDougall, Curtis (1982), Interpretative Reporting, Estados Unidos: Macmillan.

Mearsheimer, Jonh (2003), The tragedy of great power politics, Estados Unidos: Norton.

Medina-Gutiérrez, Felipe (2018), "Yemen: un escenario de guerra y crisis humanitaria", en OASIS-Observatorio de Análisis de los Sistemas Internacionales, vol. 27, Colombia: Universidad Externado de Colombia.

Meyer, David (2001), “Support Vector Machines”, en R News, vol. 1, núm. 3, Austria: R-News.

Moreno-Mercado, José Manuel (2018), "La cobertura mediática de los conflictos armados. El caso de Siria”, en Revista de Paz y Conflictos, vol. 11, núm. 2, España: Instituto de la Paz y los Conflictos.

Moreno-Mercado, José Manuel (2019), “La comunicación estratégica en RT: construcción de encuadres en los conflictos de Yemen y Ucrania", en Revista Politica y Estrategia, núm. 133, Chile: Academia Nacional de Estudios Políticos y Estrategias.

Neuman, Russel et al. (1992), Common Knowledge: News and the Construction of Political Meaning, Estados Unidos: University of Chicago Press. 
Osowski, Stanislaw et al. (2004), "MLP and SVM networks -a comparative study", en Procs of the $6^{a}$ Nordic Signal Processing Symposium (Norsig), Noruega: Norsig.

Pantti, Mervi (2016), "Seeing and not seeing the Syrian crisis: New visibility and the visual framing of the Syrian conflict in seven newspapers and their online editions", en JOMEC Journal, vol. 4, Reino Unido: Cardiff University Press.

Peña-Ramos, José Antonio y Jorquera-Mery, Constanza Fernanda (2017), "El conflicto mapuche en el canal iraní HispanTV”, en Martínez, Rafael [ed.], Comunicación politica en seguridad y defensa: España y América Latina, España: Barcelona Centre for International Affairs (CIDOB).

Ramos, Juan (2003), "Using TF-IDF to determine Word relevance in document queries”, en Proceedings of the first instructional conference on machine learning, núm. 242, Estados Unidos: Proceedings of the first instructional conference on machine learning.

Rettberg, Jill y Gajjala, Radhika (2016), “Terrorists or cowards: negative portrayals of male Syrian refugees in social media”, en Feminist Media Studies, vol. 16, núm. 1, Reino Unido: Routledge.

RT (2016), "Lo último de la escalada del conflicto en el este de Ucrania”, en RT, Rusia.

RT (2017), "Pompeo aterriza en Arabia Saudita e inmediatamente pide nuevas sanciones contra Irán”, en $R T$, Rusia.

RT (2017), "La flota rusa del mar Negro inicia ejercicios de operaciones anti sabotaje”, en $R T$, Rusia.

RT (2018), “¿Ayuda Trump a Arabia Saudita a destruir Yemen?”, en RT, Rusia.

Sahagún, Felipe (2018), “Assad, Putin y Jamenei pueden dormir tranquilos”, en El Mundo, España.

Salisbury, Peter (2015), "Yemen and the Saudi-Iranian 'Cold War”, en Research Paper, Middle East and North Africa Programme, Chatham House, the Royal Institute of International Affairs, núm. 11, Reino Unido: Chatham House.

Sánchez-de Rojas Díaz, Emilio (2018), "El 'soft power' en las guerras de información. Las operaciones de influencia de las grandes potencias”, en Bie3: Instituto Español de Estudios Estratégicos, núm. 10, España: Ministerio de Defensa.

Segovia, Carlos (2019), "Moncloa vuelve a frenar la venta de armamento a Arabia Saudí”, en El Mundo, España.

Semetko, Holli y Valkenburg, Patti (2000), "Framing European Politics: A content analysis of press and television news", en Journal of Communication, vol. 50, núm. 2, Estados Unidos: Wiley Online Library.

Sheafer, Tamir y Gabay, Italy (2009), "Mediated public diplomacy: A strategic contest over international agenda building and frame building", en Political Communication, vol. 26, núm. 4, Estados Unidos: Taylor and Francis.

Steele, Jonathan (2015), "New Cold War?", en Underwater Battlespace, Reino Unido: Spokesman Books.

Tsygankov, Andrei (2015), "Vladimir Putin's last stand: the sources of Russia's Ukraine Policy”, en Post-Soviet Affairs, vol. 31, núm. 4, Estados Unidos: Taylor and Francis.

Tzemprin, Athina et al. (2015), “The Middle East cold war: Iran-Saudi Arabia and the way ahead”, en Politička misao: časopis za politologiju, vol. 52, núm. 4-5, Serbia: Nova srpska politička misao.

United Nations and Word Bank (2018), Pathways for Peace: Inclusive Approaches to Preventing Violent Conflict. Main Messages and Emerging Policy Directions, Estados Unidos: Banco Mundial. 
Vuotto, Andrés et al. (2015), "Aplicación del factor TF-IDF en el análisis semántico de una colección documental”, en Biblios: Journal of Librarianship and Information Science, núm. 60, Estados Unidos: Sage Journals.

Wolfsfeld, Gadi (2018), "The role of the media in violent conflicts in the digital age: IsraeliPalestinian leaders' perceptions”, en Media, War \& Conflict, vol. 11, núm. 1, Estados Unidos: Sage Journals.

Yablokov, Ilya (2015), "Conspiracy theories as a Russian Public Diplomacy tool: The case of Russia Today (RT)”, en Politics, vol. 35, núm. 3-4, Estados Unidos: Sage Journals.

Yárnoz, Carlos (2018), "Barcos sin principios”, en El Pais, España.

Zabyelina, Yuliya (2019), "Vigilant justice and informal policing in post-Euromaidan Ukraine”, en Post-Soviet Affairs, Estados Unidos: Sage Journals.

\section{Anexo}

\section{Tabla 1}

Editoriales por medio y conflicto (2015-2019)

\begin{tabular}{lcccc}
\multicolumn{1}{r}{ Medio } & \multicolumn{2}{c}{ Ucrania } & \multicolumn{2}{c}{ Yemen } \\
\hline El País & $\mathrm{n}$ & $\%$ & $\mathrm{n}$ & $\%$ \\
\hline El Mundo & 285 & 21.01 & 178 & 6.14 \\
\hline RT & 149 & 10.98 & 272 & 9.39 \\
\hline HispanTV & 300 & 22.12 & 292 & 10.08 \\
\hline Total & 622 & 45.87 & 2.154 & 74.37 \\
\hline
\end{tabular}

Fuente: Elaboración propia. 


\section{Gráfico 1}

Evolución de la cobertura en el conflicto yemení (2015-2019 $\mathrm{n}$ de editoriales codificados)

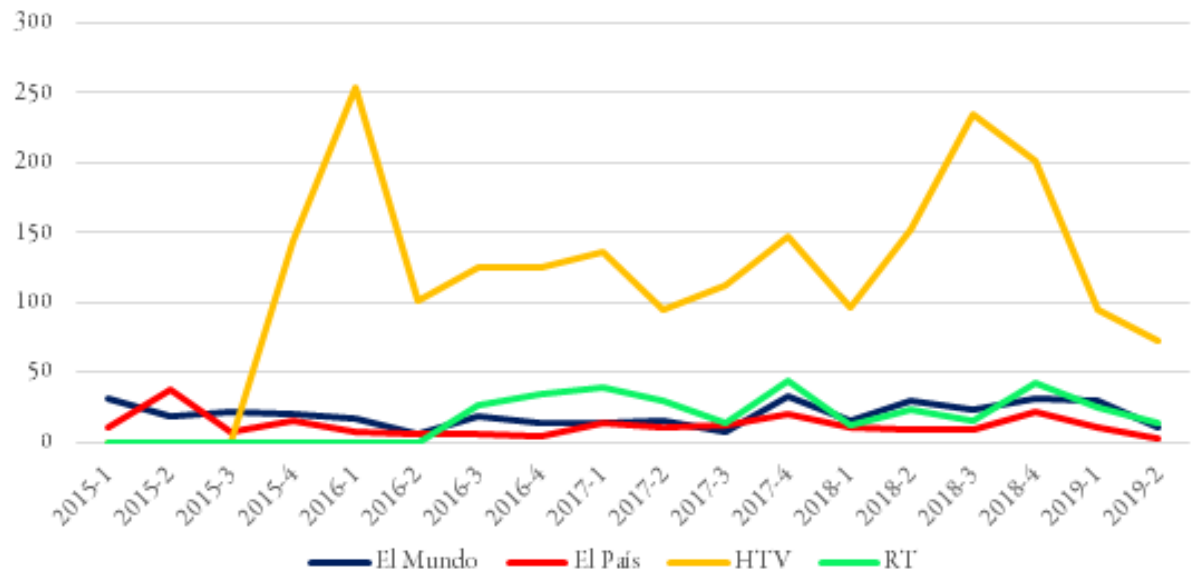

Fuente: Elaboración propia.

$$
\text { Gráfico } 2
$$

Evolución de la cobertura en el conflicto ucraniano (2015-2019, $n$ de editoriales codificados)

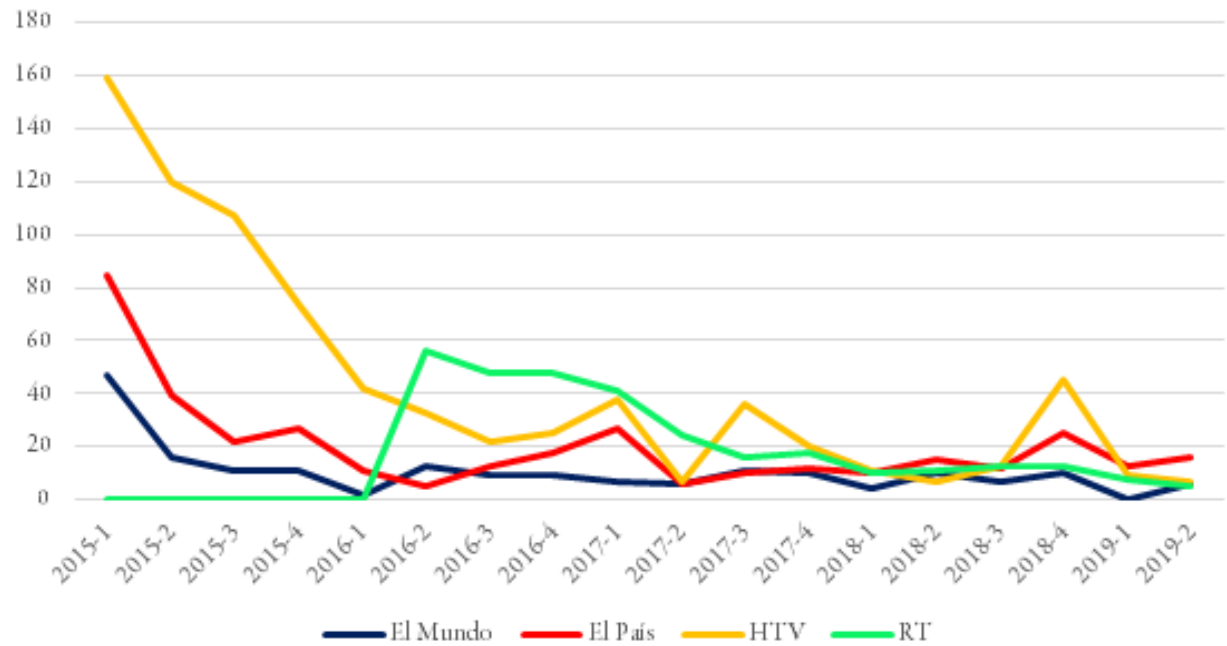

Fuente: Elaboración propia. 


\section{Gráfico 3}

Encuadres en el conflicto de Ucrania (\%)

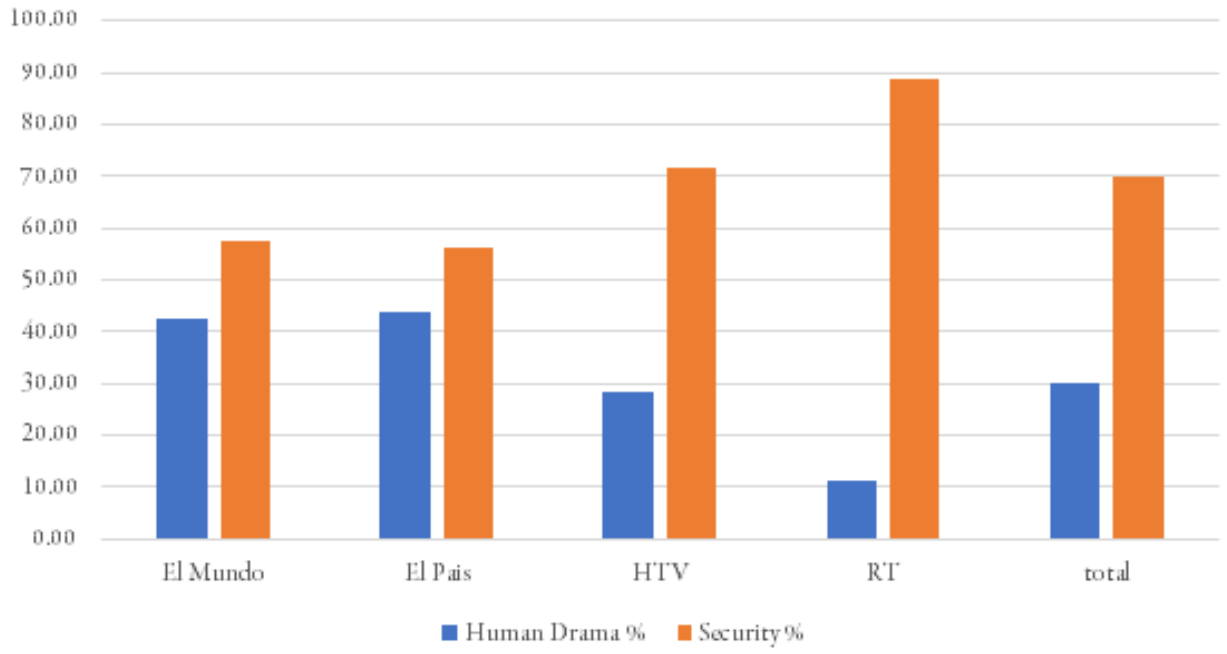

Fuente: Elaboración propia.

\section{Gráfico 4}

\section{Encuadres en el conflicto de Yemen (\%)}

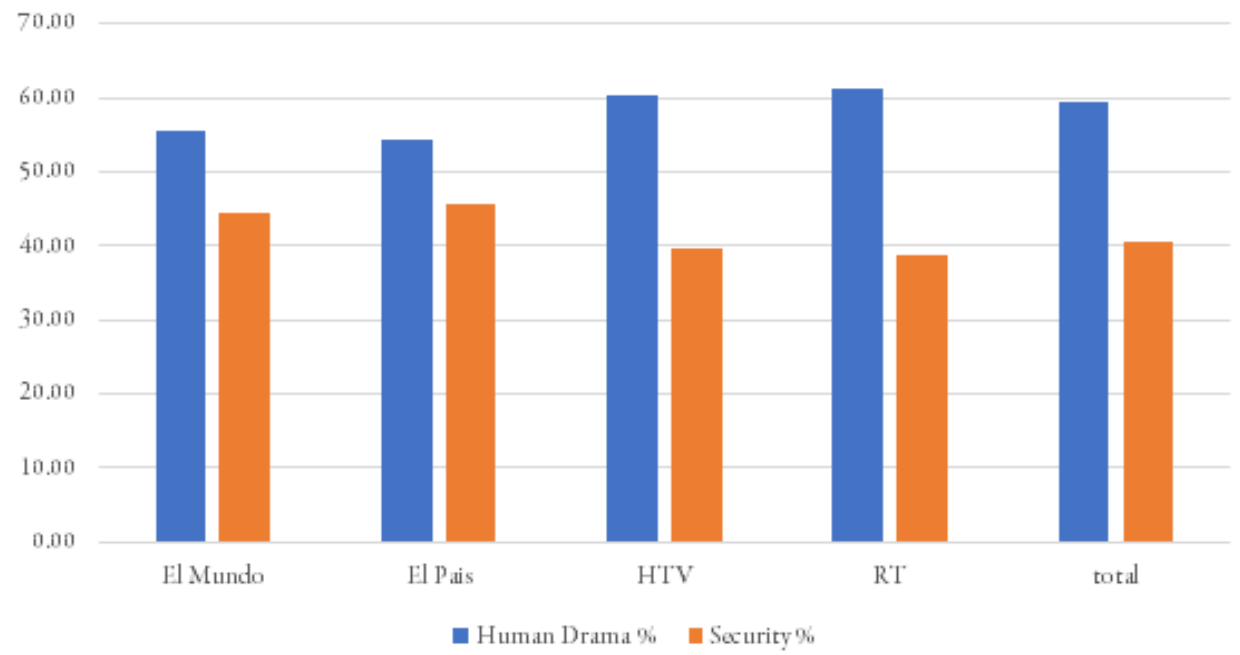

Fuente: Elaboración propia. 


\section{Gráfico 5}

\section{Comparativa de encuadres en los conflictos (\%)}

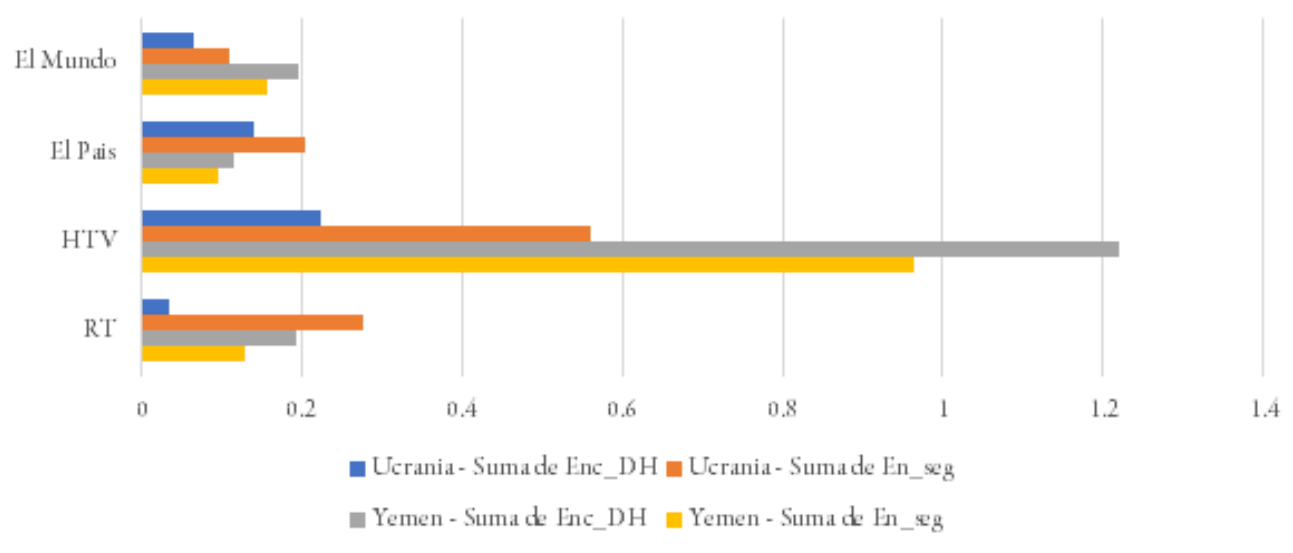

Fuente: Elaboración propia. 
José Manuel Moreno Mercado. Doctorando en el Departamento de Ciencia Política y de la Administración de la Universidad de Granada; Graduado en Ciencias Políticas y de la Administración y Magíster en Cultura de Paz, Conflictos, Educación y Derechos Humanos por la misma Universidad y el Instituto de la Paz y los Conflictos. Líneas de investigación: comunicación política, medios y conflictos, opinión pública, relaciones internacionales. Publicaciones recientes: Moreno Mercado, José Manuel y Jiménez Cabello, José Manuel (2019), "Fuerzas Armadas y medios de comunicación: la mujer como elemento modernizador", en El profesional de la información, vol. 28, núm. 2, España: Profesional de la Información; Moreno Mercado, José Manuel (2019), "La comunicación estratégica en RT: construcción de encuadres en los conflictos de Yemen y Ucrania”, en Revista Politica y Estrategia, núm. 133, Chile: Academia Nacional de Estudios Políticos y Estratégicos; Moreno Mercado, José Manuel (2018), "La cobertura mediática de los conflictos armados. El caso de Siria”, en Revista de Pazy Conflictos, vol. 11, núm. 2, España: Instituto de la Paz y los Conflictos.

Javier García Marín. Profesor en el Departamento Ciencia Política y de la Administración de la Universidad de Granada; Doctor en Ciencia Política, Magíster en Economía y Relaciones Internacionales y Licenciado en Historia Contemporánea por la Universidad Autónoma de Madrid. Líneas de investigación: comunicación política, metodología en comunicación, medios y conflictos, campañas electorales. Publicaciones recientes: García Marín, Javier y García Luengo, Óscar (2019), "Populist Discourse in the 21ST Century”, en Hidalgo Tenorio, Encarnación, Benítez Castro, Miguel Ángel y De Cesare, Francesca, Populist Discourse: Critical Approaches to Contemporany Politics, Reino Unido: Routledge; García Marín, Javier, Calatrava García, Adolfo y García Luengo, Óscar (2018), "Debates electorales y conflicto: Un análisis con máquinas de soporte virtual (SVM) de la cobertura mediática de los debates en España desde 2008", en El profesional de la información, vol. 27, núm. 3, España: El Profesional de la Información; García Marín, Javier y Calatrava García, Adolfo (2018), “The Use of Supervised Learning Algorithms in Political Communication and Media Studies: Locating Frames in the Press", en Communication \& Society, vol. 31, núm. 3, España: Universidad de Navarra. 